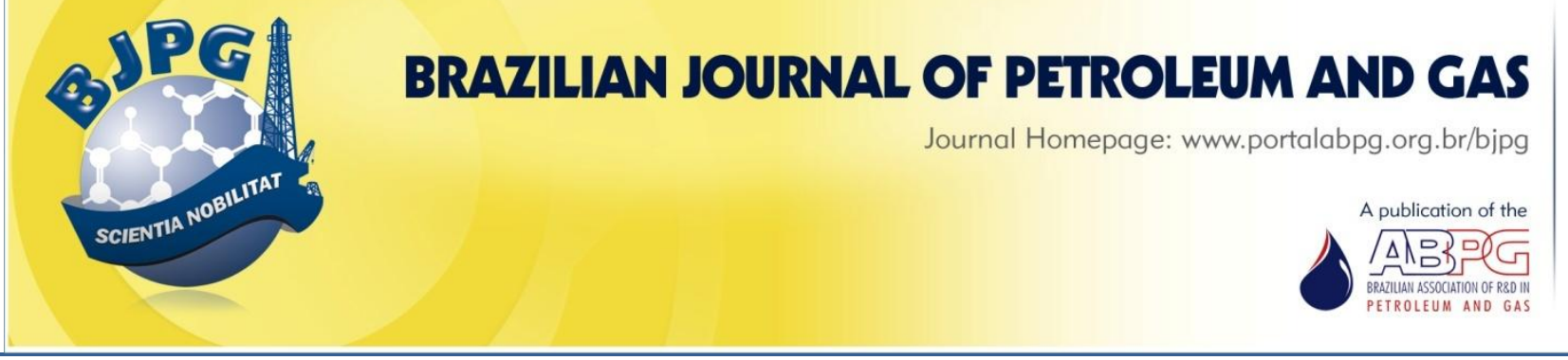

\title{
EFFECT OF DISCRETIZATION ON CALCULATION OF SLANT WELL PRODUCTIVITY INDEX IN NUMERICAL RESERVOIR SIMULATION
}

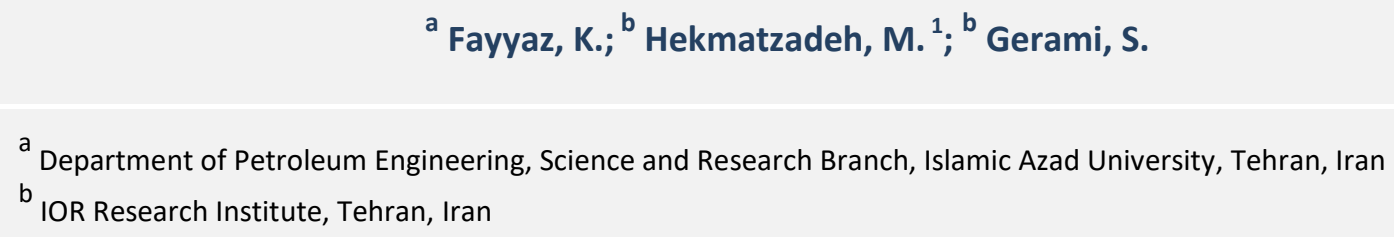

Received: 20.06.2017 / Revised: 04.09.2017 / Accepted: 04.09.2017 / Published on line: 16.10.2017

\begin{abstract}
Slant well is a non-vertical form of wells used typically for extracting oil and gas resources that cannot be accessed through traditional vertical well drilling. Deviation in these wells results in the geometric skin factor, which has a negative value and increases the productivity index. Various analytical/semi-analytical equations estimate the geometric skin factor for the slant well for slightly compressible fluids. This study attempts to show that the productivity calculated by numerical simulation for the slant well in a singlephase (oil/gas) reservoir is sensitive to grid resolution. For this purpose, first, a slant well is defined within a local grid refinement ("LGR") system. Afterwards, the slant skin factor is calculated for cases having different LGR resolution for a constant degree of inclination to find a reasonable LGR system. Then, the applicability of this optimum LGR for all deviation angles with comparison with analytical model is evaluated. This evaluation shows that optimum LGR is particular for each well deviation angle. Although some of the analytical/semi analytical models are developed for slightly compressible fluids, these models also are applicable for compressible fluids (i.e. dry gas). Furthermore, the calculated skin for the slant well in numerical simulation for optimum LGR is not sensitive to production rate or oil viscosity.
\end{abstract}

\section{KEYWORDS}

slant well; numerical simulation; local grid refinement; geometric skin

\footnotetext{
${ }^{1}$ To whom all correspondence should be addressed.

Address: Department of Petroleum Engineering, Science and Research Branch, Islamic Azad University, P. O. Box 14515/775, Tehran, Iran

e-mail: hekmatzadeh@nioc.ir

doi:10.5419/bjpg2017-0012
} 


\section{INTRODUCTION}

It is very common for wells to have a certain angle between their axis and the normal formation plane. In such wells, the trajectory is not parallel to gridlines of a Cartesian reservoir model called "slant well". Figure 1 shows the schematic of vertical and slant wells. Slant wells are used for protecting environment sensitive areas or drilling into the reservoir where vertical access is difficult or not possible (Chen et al., 1995). This directional drilling also helps to increase well productivity by increasing the contact length and decreasing the pressure drop in comparison to vertical wells.

Although such wells are usual, a limited number of studies address the performance of such completions. Roemershauser and Hawkins (1955) studied steady-state flow in a reservoir producing through a fully penetrating slant well using an electrical model. They considered a circular reservoir with finite extent and concluded that the deviation of a fully penetrating well causes an increase in the well productivity. The increase in well productivity results from the decrease in the resistance to flow around the wellbore and an increase in the producing area exposed to flow. This increase in well productivity indicates that a fully penetrating slant well creates a negative skin effect. Roemershauser and Hawkins (1955)

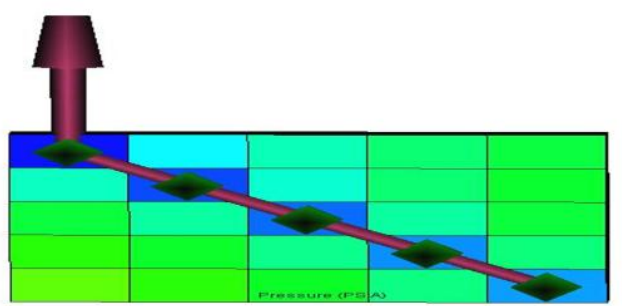

(A)

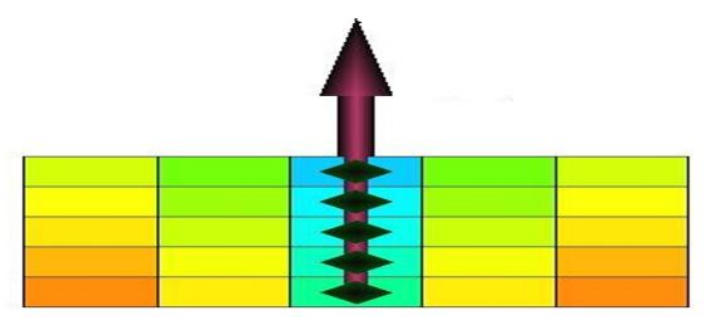

(B)

Figure 1. (A) slant well; (B) vertical well. graphed the increase in well productivity in comparison to the angle of the slant well.

Cinco et al. (1975) conducted one of the most remarkable studies of the unsteady-state performance of slant wells. They assumed a slant well in an infinite slab. The effects of top and bottom with no flow boundaries were modeled by image method. They took the point source solution to solve the ensuing diffusivity equation. They calculated the pseudo-skin factor due to well deviation from vertical direction by subtracting the dimensionless wellbore pressure of a vertical well from the one obtained for a slant one. After computing several data for geometric skin for slant wells, they introduced a correlation to compute pseudo-skin as a function of the deviation angle and dimensionless formation thickness as is shown in Equations (1) to (3).

$\mathrm{S}_{\theta}=-\left(\frac{\hat{\theta}_{\mathrm{w}}}{41}\right)^{2.06}-\left(\frac{\hat{\theta}_{\mathrm{w}}}{56}\right)^{1.865} \times \log \left(\frac{\mathrm{h}_{\mathrm{D}}}{100}\right)$

For $0^{\circ} \leq \dot{\theta}_{\mathrm{w}} \leq 75^{\circ}$

$\mathrm{h}_{\mathrm{D}}=\frac{\mathrm{h}}{\mathrm{r}_{\mathrm{w}}} \sqrt{\frac{\mathrm{k}_{\mathrm{h}}}{\mathrm{k}_{\mathrm{v}}}}$

$\dot{\theta}_{\mathrm{w}}=\tan ^{-1}\left(\sqrt{\frac{\mathrm{k}_{\mathrm{v}}}{\mathrm{k}_{\mathrm{h}}}} \tan \theta_{\mathrm{w}}\right)$

This correlation is only applicable for fully penetrating slant wells with angle less than 75 degrees. Although trying to incorporate the anisotropy effect into their model, Rogers and Economides (1996) claimed that it is only applicable in isotropic mediums (in isotropic mediums $k_{v}=k_{h}$, in anisotropic mediums $k_{h}>k_{v}$ ).

Van Der Vlis et al. (1979) used an electrolytic analogue model to develop concept of effective wellbore radius for slant wells as is shown in Eq. (4). One should note that this model also is applicable only applicable for isotropic reservoirs.

$r_{w e f f}=\left(\frac{h_{o}}{4 \cos \theta}\right)\left[0.454 \sin \left(360\left(\frac{r_{w}}{h_{o}}\right)\right)\right]^{\cos \theta}$

$\mathrm{S}_{\theta}=-\ln \left(\frac{\mathrm{r}_{\mathrm{w}, \mathrm{eff}}}{\mathrm{r}_{\mathrm{w}}}\right)$ 
Later, Besson (1990) obtained pressure decline curves for a set of slant wells in homogeneous, isotropic and infinite system by using a semianalytical simulator.

$\mathrm{S}_{\theta}=\ln \left(\frac{4 \mathrm{r}_{\mathrm{w}}}{\mathrm{L}}\right)+\frac{\mathrm{H}}{\mathrm{L}} \ln \left(\frac{\sqrt{\mathrm{LH}}}{4 \mathrm{r}_{\mathrm{w}}}\right)$

Then, the above equation is transformed for an anisotropic reservoir through a spatial transformation from real medium into equivalent isotropic medium.

$\mathrm{S}_{\theta}=\ln \left(\frac{4 \mathrm{r}_{\mathrm{w}}}{\mathrm{L}} \frac{1}{\mathrm{I}_{\mathrm{ani}} \gamma}\right)+\frac{\mathrm{H}}{\gamma \mathrm{L}} \ln \left(\frac{\sqrt{\mathrm{LH}}}{4 \mathrm{r}_{\mathrm{w}}} \frac{\mathrm{a}_{\mathrm{ani}} \sqrt{\gamma}}{1+\frac{1}{\gamma}}\right)$

$\gamma=\sqrt{\cos ^{2} \theta+\frac{1}{\mathrm{Iani}^{2}} \sin ^{2} \theta}$

$I_{\mathrm{ani}}=\sqrt{\frac{\mathrm{k}_{\mathrm{h}}}{\mathrm{k}_{\mathrm{v}}}}$

In Eq. (9), $I_{a n i}$ is called anisotropic index. It should be noted that in most of the cases $I_{\text {ani }}$ is greater than 1, because of bed deposition of sediments. Rogers and Economides (1996) developed a correlation for the geometric skin factor of fully penetrating slant wells in anisotropic condition as is shown in Eq. (10) and Eq. (11).

$\mathrm{I}_{\mathrm{ani}}<1 ; \mathrm{S}_{\theta}=-1.64 \frac{\sin \theta^{1.77} \mathrm{~h}_{\mathrm{D}}^{0.184}}{\mathrm{I}_{\mathrm{ani}}^{0.821}}$

$\mathrm{I}_{\mathrm{ani}} \geq 1 ; \mathrm{S}_{\theta}=-2.48 \frac{\sin \theta^{5.87} \mathrm{~h}_{\mathrm{D}}^{0.152}}{\mathrm{I}_{\mathrm{ani}}{ }^{0.964}}$

Suk Kyoon et al. (2008) conducted a sensitivity analysis of the available analytical models developed for calculating the productivity of slant wells. They claimed that these correlations were inappropriate for extremely deviated wells; namely, the ones with deviation angle over 75 degrees.

Haijing et al. (2012) presented an inflow model for a highly deviated well in an anisotropic reservoir and concluded that the increase in anisotropy index resulted in an increase in the effect of the deviation angle on well productivity.

By using a sensitivity study, Ghahri and Jamiolahmady (2012) claimed that the skin values obtained from Rogers and Economides (1996) were under estimated and significantly different from those predicted by equations proposed by Besson (1990) and Cinco et al (1975) using the same transformation for isotropic media.

Based on previous sensitivity studies, in this work, the results of the Cinco et al. (1975) analytical model for isotropic reservoir and the Rogers and Economides (1996) for anisotropic one are considered as a basis for comparison with computed slant skin from numerical simulation to understand the discretization effect. Then, a numerical simulation with specifications similar to Cinco et al. (1975) model assumptions has been developed as a base case.

The model includes a slant well with 45 or 75 degree deviation (the most extreme degree of deviation for slant wells in Cinco et al.'s study) in a homogeneous (isotropic and anisotropic) reservoirs with Cartesian grids. All simulations were done with a single-phase flow (dry gas or undersaturated oil) in a closed (no-flow) boundary reservoir. By defining slant well within a LGR system, the goal was to show the sensitivity of black oil simulation results to the well production rate, as well as the resolution of the local grid opted for modeling the slant well of any deviation angle. Then, to deal with the LGR sensitivity problem, the authors suggest finding an optimum LGR system for each simulation case by taking the results of Cinco et al. (1975) as basis.

This paper proceeds as follows: first, the details of the numerical simulation methodology are illustrated. Then, simulation results are presented and explained. Finally, the conclusions end the paper.

\section{METHODOLOGY}

To simulate Cinco et al.'s (1975) analytical model, a base case with single-phase fluid (either slightly-compressible or compressible) is considered. The rock and fluid parameters for a single-phased oil and dry gas reservoirs are shown in Table 1. The Cartesian coordinate is chosen to simulate slant wells. The reservoir is $34800 \mathrm{ft} \times$ $34800 \mathrm{ft} \times 100 \mathrm{ft}$, with a global grid size of $201 \times$ $201 \times 1$. 
Table 1. Single phase fluid reservoir properties.

\begin{tabular}{ccc}
\hline Parameters & Values \\
\hline Initial pressure & Oil & $1000 \mathrm{psi}$ \\
production rate & Gas & $2000 \mathrm{M} \mathrm{scf} / \mathrm{day}$ \\
Permeability(isotropic) & \multicolumn{2}{c}{$10 \mathrm{md}$} \\
Porosity & 0.2 \\
Oil viscosity & Oil & $2 \mathrm{cp}$ \\
Formation compressibility & Gas & $0.0244 \mathrm{cp}$ \\
compressibility & \multicolumn{2}{c}{$6.66 \mathrm{E}-06 \mathrm{psi}^{-1}$} \\
Reservoir depth & Oil & $3 \mathrm{E}-06 \mathrm{psi}^{-1}$ \\
Reservoir thickness & Gas & $1.217 \mathrm{E}-04 \mathrm{psi}^{-1}$ \\
Wellbore radius & \multicolumn{2}{c}{$5000 \mathrm{ft}$} \\
Oil formation volume & \multicolumn{2}{c}{$100 \mathrm{ft}$} \\
factor & \multicolumn{2}{c}{$0.5 \mathrm{ft}$} \\
Critical gas temperature & \multicolumn{2}{c}{$1.05 \mathrm{bbl} / \mathrm{stb}^{2}$} \\
Critical gas pressure & $343.1{ }^{\circ} \mathrm{R}$ \\
\end{tabular}

In this research, the deviations of the well from vertical axis for the base case are 75 and 45 degrees. Therefore, the grid sizes selected are equal to $373.2 \times 373.2 \times 100 \mathrm{ft}$ and $100 \times 100 \times 100 \mathrm{ft}$ respectively to pass from the grid center as is shown in Fig. 2.

Then, the bottom-hole pressure versus time as a

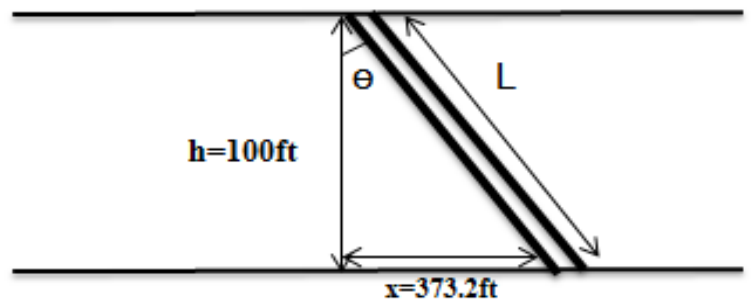

(A)

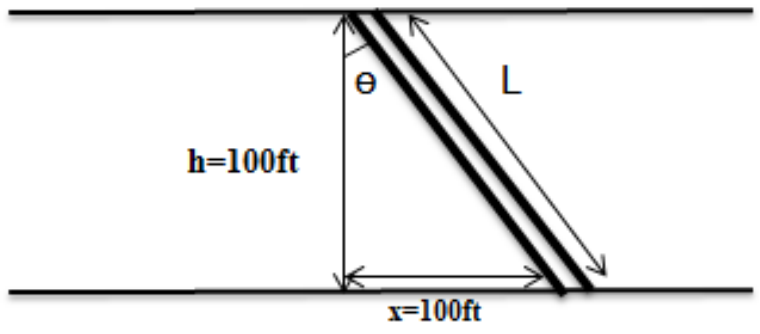

(B) numerical simulation output is analyzed using a straight-line technique. After that, the skin factor is calculated by Eq. (12) and Eq. (13) for oil and gas well respectively (Van Everdingen and Hurst, 1994);

$$
\mathrm{S}=1.151\left[\frac{\mathrm{p}_{\mathrm{i}}-\mathrm{p}_{1 \mathrm{hr}}}{|\mathrm{m}|}-\log \left(\frac{\mathrm{k}}{\phi \mu \mathrm{c}_{\mathrm{t}} \mathrm{r}_{\mathrm{w}}^{2}}\right)+3.23\right]
$$

$\mathrm{S}=1.151\left[\frac{\Delta \Psi}{\mathrm{m}}-\log \left(\frac{\mathrm{k}}{\phi \mu_{\mathrm{gi}} \mathrm{c}_{\mathrm{i}} \mathrm{r}_{\mathrm{w}}^{2}}\right)+3.23\right]$

The values of damage skin, partial penetration skin, and anisotropy skin are considered zero in numerical simulation. Hence, according to Eq. (14), the total skin factor obtained from semi-log analysis is equal to the geometric skin factor. For the gas well, the dependent skin rate is also calculated and subtracted from total skin to obtain geometric skin factor as is shown in Eq. (15), (Wattenbarger \& Ramey, 1986).

$$
\begin{aligned}
& \mathrm{S}=\mathrm{S}_{d}+\mathrm{S}_{\mathrm{p}}+\mathrm{S}_{\mathrm{ani}}+\mathrm{S}_{\Theta} \\
& \mathrm{S}^{\prime}=\mathrm{S}+\mathrm{Dq}
\end{aligned}
$$

Therefore, it is possible to perform a comparison between a numerical geometric skin and an analytical/semi analytical one.

Figure 2. Slant well with different angles; (A) 75 degree, (B) 45 degree. 


\section{DISCUSSION OF THE NUMERICAL SIMULATION RESULTS}

Table 2 shows the comparison between the numerical geometric and the analytical skin calculated by different models for the base case for oil and gas well. It also shows that the geometric skin factor of the numerical model in base case for oil and gas reservoirs do not present a significant difference with each other but their value is different from analytical model. To improve the accuracy of the numerical model, the grid sizes that slant well passes from must be reduced using the LGR approach. The implementation of LGR for a slant well in a Cartesian reservoir model should be done according to a specific pattern (Farokhi et al., 2015). Hence, the grid sizes are fined in the way that the number of local grids in $x$ and $z$ directions be equal, so that the slant well with any angle passes from center of grid blocks as it is shown in the third and fourth columns of Table 3 .

Table 3 indicates that, by reducing the grid sizes, the numerical model results become closer to the ones presented by the analytical model. Thus, optimal grid sizes for 75 and 45-degree slanted wells in oil and gas reservoirs are obtained by applying two restrictions as follows:

1) The difference between numerical and analytical model results should be in acceptable range of error \pm 0.5 ;

2) A reduction in grid sizes to less than optimum sizes does not cause significant changes in the results of the numerical model.

Therefore, the optimum grid sizes for 75 and 45-degree slanted wells in both oil and gas reservoirs are $9.33 \mathrm{ft} \times 9.33 \mathrm{ft} \times 2.5 \mathrm{ft}$ and $2.5 \mathrm{ft} \times 2.5 \times 2.5 \mathrm{ft}$, respectively. One should note that the optimum LGR for both oil and gas reservoirs are the same. But, different LGR systems should be considered for different deviation angles.

After finding the optimum grid size, sensitivity analyses on production rate, oil viscosity, and anisotropy ratio are done to find out how they affect the geometric skin factor obtained by numerical simulation.

Table 2. Comparison of geometric skin calculated by analytical and numerical models in oil and gas reservoirs for the base case.

\begin{tabular}{cccc}
\hline $\begin{array}{c}\text { Angle } \\
\text { (Degree) }\end{array}$ & \multicolumn{2}{c}{ Numerical Model } & Analytical \\
Oil & Gas & Models \\
\hline 75 & -2.44 & $-\mathbf{3 . 0 7 1}$ & -3.98 \\
45 & -0.68 & -0.697 & -1.41 \\
\hline
\end{tabular}

Table 3. Comparison of geometric skin values for different grid size in slant well in oil and gas reservoirs.

\begin{tabular}{|c|c|c|c|c|c|c|c|c|}
\hline \multirow{2}{*}{ Fluid } & \multirow{2}{*}{$\begin{array}{c}\text { Angle } \\
\text { (Degree) }\end{array}$} & \multirow{2}{*}{$\begin{array}{c}\text { Grid size } \\
\text { (ft) }\end{array}$} & \multirow{2}{*}{$\begin{array}{l}\text { Number } \\
\text { of grids }\end{array}$} & \multicolumn{2}{|c|}{ Numerical model skin } & \multirow{2}{*}{$\begin{array}{c}\text { Analytical } \\
\text { Model }\end{array}$} & \multicolumn{2}{|c|}{ Difference } \\
\hline & & & & Oil & Gas & & Oil & Gas \\
\hline \multirow{8}{*}{ Oil } & \multirow{4}{*}{75} & $74.64 \times 20$ & $5 \times 5$ & -2.44 & -3.07 & \multirow{4}{*}{-3.98} & 1.54 & 0.910 \\
\hline & & $37.32 \times 10$ & $10 \times 10$ & -2.66 & -3.202 & & 1.32 & 0.778 \\
\hline & & $9.33 \times 2.5$ & $40 \times 40$ & -3.47 & -3.993 & & 0.51 & 0.013 \\
\hline & & $3.732 \times 1$ & $\begin{array}{c}100 \times \\
100\end{array}$ & -3.5 & -4.01 & & 0.48 & 0.030 \\
\hline & \multirow{4}{*}{45} & $20 \times 20$ & $5 \times 5$ & -0.68 & -0.69 & \multirow{4}{*}{-1.41} & 0.730 & 0.720 \\
\hline & & $10 \times 10$ & $10 \times 10$ & -0.74 & -0.881 & & 0.670 & 0.529 \\
\hline & & $2.5 \times 2.5$ & $40 \times 40$ & -1.179 & -1.305 & & 0.231 & 0.105 \\
\hline & & $1 \times 1$ & $\begin{array}{c}100 \times \\
100\end{array}$ & -1.17 & -1.349 & & 0.240 & 0.061 \\
\hline
\end{tabular}


Table 4. Geometric skin factor in different oil production rate for slant well.

\begin{tabular}{ccc}
\hline $\begin{array}{c}\text { Angle } \\
\text { (Degree) }\end{array}$ & $\begin{array}{c}\mathbf{Q}_{\text {oil }} \\
\text { (bbl/day) }\end{array}$ & $\begin{array}{c}\text { Skin } \\
\text { Value }\end{array}$ \\
\hline \multirow{3}{*}{75} & 75 & -3.468 \\
& 100 & -3.47 \\
& 200 & -3.40 \\
\hline \multirow{2}{*}{45} & 75 & -1.176 \\
& 100 & -1.179 \\
& 200 & -1.174 \\
\hline
\end{tabular}

Table 5. Geometric skin factor in different gas production rate for slant well.

\begin{tabular}{ccc}
\hline $\begin{array}{c}\text { Angle } \\
\text { (Degree) }\end{array}$ & $\begin{array}{c}\mathbf{Q}_{\text {gas }} \\
\text { (Mscf/day) }\end{array}$ & Skin Value \\
\hline \multirow{2}{*}{75} & 1500 & -3.992 \\
& 2000 & -3.993 \\
& 2500 & -3.999 \\
\hline \multirow{2}{*}{45} & 1500 & -1.41 \\
& 2000 & -1.305 \\
& 2500 & -1.38 \\
\hline
\end{tabular}

Table 6. Geometric skin factor in different oil viscosity values for slant well.

\begin{tabular}{ccc}
\hline $\begin{array}{c}\text { Angle } \\
\text { (Degree) }\end{array}$ & $\begin{array}{c}\text { Viscosity } \\
\text { (cp) }\end{array}$ & skin value \\
\hline \multirow{3}{*}{75} & 1 & -3.477 \\
& 2 & -3.47 \\
& 3 & -3.40 \\
\hline \multirow{2}{*}{45} & 1 & -1.179 \\
& 2 & -1.179 \\
& 3 & -1.173 \\
\hline
\end{tabular}

\subsection{Production rate}

Tables 4 and 5 show that changing the production rate to optimal grid size for oil and gas well does not affect the geometric skin factor.

\subsection{Oil viscosity}

Table 6 shows that changing the viscosity for optimum grid size for oil well does not affect the geometric skin factor.

\subsection{Anisotropy ratio}

In anisotropic reservoirs, by neglecting the other parameters of skin factor, the total skin factor is equal to the sum of geometric skin factor and anisotropy skin factor, as is shown in Eq. (16).
Hence, the geometric skin factor is obtained by subtracting the anisotropy skin factor from the total skin factor. The anisotropy skin factor is calculated according to Eq. (17) (Abbaszadeh \& Hegeman, 1990).

$\mathrm{S}_{\mathrm{t}}=\mathrm{S}_{\theta}+\mathrm{S}_{\mathrm{ani}}$

$\mathrm{S}_{\mathrm{ani}}=-\ln \frac{1+\frac{1}{\sqrt{\cos ^{2} \theta_{\mathrm{w}}+\frac{\mathrm{k}_{\mathrm{v}}}{\mathrm{k}_{\mathrm{h}}} \sin ^{2} \theta_{\mathrm{w}}}}}{2}$

Table 7 shows the values of geometric skin for different anisotropy ratio for oil and gas reservoirs. The values calculated are compared also with analytical model. 
Table 7. Comparison of geometric skin factor values in various anisotropy ratio for slanted well in anisotropic reservoirs.

\begin{tabular}{|c|c|c|c|c|c|c|c|c|c|c|}
\hline \multirow{2}{*}{$\begin{array}{c}\text { Angle } \\
\text { (Degree) }\end{array}$} & \multirow{2}{*}{$K_{v}$} & \multirow[t]{2}{*}{$I_{\text {ani }}$} & \multicolumn{2}{|c|}{$\mathrm{S}_{\mathrm{t}}$} & \multirow{2}{*}{$\mathrm{S}_{\mathrm{ani}}$} & \multicolumn{2}{|c|}{$\begin{array}{c}\mathrm{S}_{\theta}, \text { Numerical } \\
\text { Model }\end{array}$} & \multirow[t]{2}{*}{$\mathrm{S}_{\theta}$, Analytical Model } & \multicolumn{2}{|c|}{ Difference } \\
\hline & & & Oil & Gas & & Oil & Gas & & Oil & Gas \\
\hline \multirow{3}{*}{75} & $k_{v}=k_{h}$ & 1 & -3.47 & -3.993 & 0 & -3.47 & -3.993 & -4.52 & 1.05 & 0.527 \\
\hline & $k_{v}=0.5 k_{h}$ & 2 & -2.69 & -2.75 & -0.34 & -2.35 & -2.41 & -2.32 & 0.03 & 0.09 \\
\hline & $k_{v}=0.1 k_{h}$ & 3 & -2.09 & -2.15 & -0.53 & -1.56 & -1.62 & -1.57 & 0.01 & 0.05 \\
\hline \multirow{3}{*}{45} & $k_{v}=k_{h}$ & 1 & -1.179 & -1.305 & 0 & -1.179 & -1.305 & -0.68 & 0.499 & 0.625 \\
\hline & $k_{v}=0.5 k_{h}$ & 2 & -0.491 & -0.504 & -0.124 & -0.367 & -0.38 & -0.37 & 0.003 & 0.010 \\
\hline & $k_{v}=0.1 k_{h}$ & 3 & -0.402 & -0.412 & -0.157 & -0.245 & -0.255 & -0.251 & 0.006 & 0.004 \\
\hline
\end{tabular}

The results of Table 7 indicate that an increase in $I_{\text {ani }}$ (which means decrease in $k_{v}$ ) leads to a decrease in geometric skin. Hence, an increase in anisotropy results in a decrease in slant well productivity. The difference between calculated geometric skin from analytical and numerical model rises with an increase in well deviation angle, but it decreases with an increase in $I_{a n i}$.

\section{CONCLUSION}

Based on this study, one can observe the following results:

- Calculated geometric skins from analytical and numerical models are nearly the same for slightly compressible (oil) and compressible (dry gas) fluids;

- The determination of reservoir grid sizes for calculating geometric skin factor and, hence, productivity index in slant well requires a specific pattern. By decreasing the grid sizes, results' accuracy increase. However, decreasing the grid size to less than optimum leads to an increase in numerical simulation run time. Also, changes in the results are not sensible;

- Optimal grid sizes are unique for each well deviation angle. But, they are independent from production rate or fluid viscosity;

- An increase in reservoir anisotropy results in a decrease in slant well productivity and computed geometric skin factor;
- The authors suggest to further study the results of numerical simulations for horizontal well, slant well in radial configurations, slant wells with deviation angles greater than 75 , especially in gas condensate reservoirs.

\section{NOMENCLATURE}

$C_{t}=$ total compressibility, $\mathrm{psi}^{-1}$

$D q=$ rate dependent skin

$h, h_{o}=$ formation thicknes, $\mathrm{ft}$

$h_{D}=$ dimensionless formation thickness, $\left(h / r_{w}\right) \sqrt{k_{h} / k_{v}}$, dimensionless

$I_{\text {ani }}=$ anisotropy index, $\sqrt{k_{h} / k_{v}}$, dimensionless

$k=$ reservoir permeability, md

$k_{h}=$ reservoir permeability in horizontal direction, $m d$

$k_{v}=$ reservoir permeability in vertical direction, $m d$

$L=$ well length, $f t$

$L G R=$ local grid refinement

$m=$ slope of linear portion of semi log plot of pressure transient data, (psi/cycle)

$p_{i}=$ initial equilibrium pressure, $p s i$

$q_{o}=$ oil production rate, $s t b /$ day

$q_{g}=$ gas production rate, $s c f / d a y$

$r_{w}=$ wellbore radius, $f t$

$r_{w, \text { eff }}=$ effective wellbore radius, $f t$

$s, s_{t}=$ total skin factor

$s_{a n i}=$ anisotropy skin

$s_{p}=$ partial penetration skin

$s_{d}=$ damage skin

$S_{\vartheta}=$ slant skin due to well deviation, dimensionless

$z=$ vertical coordinate

$\phi=$ reservoir porosity, fraction

$\mu_{o}=$ oil viscosity, $c p$

$\theta=$ well deviation angle, degree

$\psi=$ pseudo pressure, $p s i^{2} / c p$ 


\section{REFERENCES}

Abbaszadeh, M.; \& Hegeman, P. S. Pressuretransient analysis for a slanted well in a reservoir with vertical pressure support. SPE Formation Evaluation, v. 5(03), p. 277-284, 1990.

https://doi.org/10.2118/19045-PA

Besson, J. Performance of slanted and horizontal wells on an anisotropic medium. In: European Petroleum Conference. Society of Petroleum Engineers, SPE-20965-MS, The Hague, Netherlands, 1990. https://doi.org/10.2118/20965-MS

Chen, G.; Tehrani, D. H.; Peden, J. M. Calculation of well productivity in a reservoir simulator (I). In: SPE Reservoir Simulation Symposium. Society of Petroleum Engineers, SPE-29121-MS, San Antonio, Texas, 1995. https://doi.org/10.2118/29121-MS

Suk Kyoon, C.; Ouyang, L. B.; Huang, W. S. A comprehensive comparative study on analytical PI/IPR correlations. In: SPE Annual Technical Conference and Exhibition. Society of Petroleum Engineers, SPE-116580-MS, San Antonio, Texas, 2008. https://doi.org/10.2118/116580-MS

Cinco, H.; Miller, F. G.; Ramey Jr, H. J. Unsteadystate pressure distribution created by a directionally drilled well. Journal of Petroleum Technology, v. 27(11), p. 1392-1400, 1975. https://doi.org/10.2118/5131-PA

Ghahri, P.; Jamiolahmady, M. A new, accurate and simple model for calculation of productivity of deviated and highly deviated well-Part I: Singlephase incompressible and compressible fluid. Fuel, v. 97, p. 24-37, 2012.

https://doi.org/10.1016/i.fuel.2012.03.030

Haijing, W.; Shifeng, X.; Cunfa, G.; Xinghua, T. O. N. G. Inflow performance for highly deviated wells in anisotropic reservoirs. Petroleum Exploration and Development, v. 39(2), p. 239-244, 2012. https://doi.org/10.1016/S1876-3804(12)60037-8
Farokhi, V.; Hekmatzadeh, M.; Gholizadeh, M.; Gerami, S. Uncertainties of Defining Slanted Wells in Black Oil Simulation. In: 77th EAGE Conference and Exhibition 2015, Madrid, Spain, 2015. https://doi.org/10.3997/2214-4609.201412794

Roemershauser, A. E.; Hawkins Jr, M. F. The effect of slant hole, drainhole, and lateral hole drilling on well productivity. Journal of Petroleum Technology, v. 7(02), p. 11-14, 1955.

https://doi.org/10.2118/437-G

Rogers, E. J.; Economides, M. J. The skin due to slant of deviated wells in permeability-anisotropic reservoirs. In: International Conference on Horizontal Well Technology. Society of Petroleum Engineers, SPE-37068-MS, Calgary, Alberta, Canada, 1996. https://doi.org/10.2118/37068-MS

Van Der Vlis, A. C.; Duns, H.; Fernandez Luque, R. Increasing well productivity in tight Chalk reservoirs. In: 10th World Petroleum Congress, WPC-18209, Bucharest, Romania, PD 7(3), p. 71-78, 1979.

Van Everdingen, A. F.; Hurst, W. The application of the Laplace transformation to flow problems in reservoirs. Journal of Petroleum Technology, v. 1(12), p. 305-324, 1949. https://doi.org/10.2118/949305-G

Wattenbarger, R. A.; Ramey Jr, H. J. Gas well testing with turbulence, damage and wellbore storage. Journal of Petroleum Technology, v. 20(08), p. 877-887, 1968.

https://doi.org/10.2118/1835-PA 